\title{
A CDM-based failure model for predicting strength of notched composite laminates
}

\author{
Ren-Horng Maa, Jung-Ho Cheng* \\ Department of Mechanical Engineering, National Taiwan University, No. 1, Sec. 4, Roosevelt Road, 10617 Taipei, Taiwan, ROC
}

Received 2 December 2000; accepted 2 April 2002

\begin{abstract}
This paper investigates the ultimate tensile failure strength of laminated composites containing a central circular hole. Based on continuum damage mechanics, a Principal Damage Model is developed by combining the generalized standard material model with the Principal Damage concept of composite materials. Three in-plane failure modes: fiber breakage, matrix cracking, and fiber/matrix interface debonding are included in the present model. After obtaining material constants and damage relations from standard tensile tests, the material constitutive relations with damage model are implemented into commercial finite element code, ABAQUS. By comparing the predicted results with the experimental data, the proposed model has proven to be capable of predicting failure strength and load-deflection relations of notched laminated composites. The effects of hole size and specimen width are discussed in detail. In addition, the advantage of the present model is demonstrated through comparison with other existing models. (C) 2002 Elsevier Science Ltd. All rights reserved.
\end{abstract}

Keywords: A. Laminates; B. Fracture; C. Damage mechanics; C. Finite element analysis; Principal Damage

\section{Introduction}

Fiber reinforced composite materials offer enormous weight saving without sacrificing strength, and are used in a wide number of engineering applications, ranging from sports goods to advanced aerospace structures. In these structural components, notches are inevitable and they tend to decrease the strength of the composite laminates. Local damage mechanisms may be initiated at an early loading stage and propagate as the load increases. The type and the accumulation of damage significantly affect the strength, failure modes, and performance of the composites. Therefore, the knowledge of damage tolerance of laminated composites containing notches is very crucial in practice for the design of load-bearing structural components made of composite materials.

Various criteria for predicting strength of composite laminates with stress concentration have been brought out over the past three decades. Whitney and Nuismer [1] proposed the point stress criterion (PSC) in 1974. It postulates that as the stress level at a characteristic

\footnotetext{
* Corresponding author. Fax: + 886-223-631-755.

E-mail addresses: jhcheng@ccms.ntu.edu.tw (J.H. Cheng), rhmaa@hotmail.com (R.H. Maa).
}

distance ahead of the notch tip reaches its critical value, the laminate is assumed to be failed. Nuismer and Whitney [2] latter changed the point stress value to the average stress over some fixed distance ahead of the notch tip, which was termed the average stress criterion (ASC). However, the characteristic length is dependent on the laminate lay-up configuration and can only be determined empirically through curve-fitting of experimental data. Backlund and Aronsson [3] proposed a damage zone model (DZM) to investigate the strength of laminates with holes of various shapes and sizes. The damage zone, ahead of the stress concentration, is approximated by a crack with cohesive stresses acting on its surfaces. Damage in the material is taken into account by reducing the cohesive stresses with increased crack opening, which in turn corresponds to increased separation of the material. However, the relation between cohesive stresses and crack opening displacement in the damage zone is much more complex when different kinds of failure modes are taken into account. Similar to the DZM, Eriksson and Aronsson [4] proposed a damage zone criterion (DZC) and AfaghiKhatibi et al. [5] proposed an effective crack growth model (ECGM) to predict the strength of laminated composites with different lay-ups. Chang and Chang [6] 
first proposed a progressive damage model (PDM) to predict tensile failure strength of laminated composites containing stress concentrations. Lamina failure criterion, stiffness degradation rules together with finite element analysis are included in the PDM analysis. Moreover, Chang and Lessard [7], Shahid and Chang [8], and Lessard and Shokrieh [9] extended the PDM to investigate compression failure strength of the laminated composites, accumulation of the damages in the laminates, and pinned-joint failure of the composites, respectively. It seems that the PDM is powerful in damage analysis and failure strength prediction. However, the development of a general accepted failure criterion and the stiffness degradation rules is still an ongoing study.

To date, hundreds of papers have been reported to deal with the failure strength of composite laminates with a hole or a crack, but few of them can correctly predict the result. The reason is that there is no unified method to describe the post-failure stiffness degradation rules of laminated composites, which is a notable shortcoming, because the post-failure behavior plays an important rule in the stress redistribution. The traditional ply discount method or partially discount method [6] may result in the underestimation of failure strength.

The authors of this article had proposed a unified model, Principal Damage Model [10], which is based on combining generalized standard material (GSM) [11] model and Principal Damage concept, and had proven to be suitable in describing damage accumulation in each ply of the laminated composites. By using the model, stiffness degradation rules after first-ply-failure can be obtained directly within the framework of irreversible thermodynamics. The uncertainty of choosing suitable stiffness degradation rules in previous researchers can be overcome by adopting the proposed model. In order to illustrate the capabilities and advantages of the model, the damage behavior of composite laminates containing a circular hole is analyzed.

\section{Statement of the problem}

Consider a laminate containing a circular hole located at the center of the plate as shown in Fig. 1. A uniform in-plane tensile loading is applied at both ends of the specimen. During loading, the plate is deformed in its own plane until it collapses at which point the plate cannot sustain any additional load. The following information will be investigated.

1. The type and size of damage in each single layer of the laminated plate as a function of applied load.

2. The in-plane load-deflection relation of the plate.

3. The ultimate tensile failure load of the plate.

\section{Continuum damage theory}

\subsection{Continuum damage mechanics}

The concept of continuum damage mechanic (CDM) was first proposed by Kachanov [12] in studying the creep rupture of metals. He found that microcracks and microcavities increase with the loading and decrease the loadcarrying ability, and used a scalar parameter, continuity factor, to describe the material degradation behavior. The marvelous idea is a challenge to the old material mechanics concept 'perfect' and 'failure', and points out that the intermediate stage exists between perfect and final rupture.

Lemaitre [13] extended Kachanov's concept and proposed the principle of strain equivalence in 1971. It suggests that any constitutive equation for a damaged material may be derived in the same way as for a virgin material except that the usual stress is replaced by the effective stress. Based on the principle, the damage variable $d$ is defined as $d=$ $\left(E^{0}-E\right) / E^{0}$, where $E^{0}$ is the stiffness of the undamaged material and $E$ the stiffness of the damaged material. Moreover, the scalar $d$ is bounded between 0 and 1, i.e. $0 \leq d \leq 1$; where $d=0$ represents perfect state and $d=1$ represents final rupture.

Later, CDM was extended to incorporate irreversible thermodynamics and the internal state variable theory. Numerous damage variable definitions, such as scalar, double scalars, second order tensor, and forth order tensor, together with damage evolution rules were developed to model fatigue, creep, creep-fatigue interaction [13], ductile plastic damage [13,14], and brittle materials such as concrete [15] and composites [16].

\subsection{Classification of damage mechanisms of laminated composites}

Damage modes of the laminated composites can be classified mainly as: fiber breakage, matrix cracking, fiber/matrix debonding, and delamination. Each associated damage mode is assumed to occur when each stress or strain components in the material principal coordinate reaches a critical value. In other words, damage in a particular mode is defined when the stress or strain components attain the damage surface. In this study, three in-plane failure modes will be discussed and incorporated in the present damage theory with a phenomenological framework, while delamination, i.e. the out-of-plane damage mode, which is irrelevant, is left out in this study. Details of the in-plane failure modes and the interrelated studies are discussed in the following sections.

\subsubsection{Fiber breakage}

Fiber rupture is primarily caused by tensile stress $\sigma_{11}$ in the fiber direction. Tensile test of 0-degree laminate shows a linear stress-strain relation up to rupture at the critical load. 


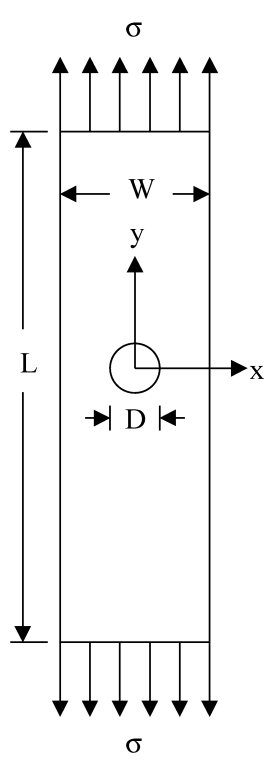

Fig. 1. Geometry of the laminate containing a central circular hole.

Thus damage mode in fiber direction is assumed to be perfect brittle failure [10].

\subsubsection{Matrix cracking}

Reifsnider [17] studied the effect of transverse crack density on stiffness reduction of 0/90 laminated plates under uniaxial quasi-static loading and cyclic loading. He found that the outer and the inner 90-degree plies of the multidirectional laminate, unlike 90-degree unidirectional laminate that fails in a catastrophic way, could still sustain part of the loading after first crack initiation. Transverse crack density in the 90-degree plies increases with the loading until the saturation level, the characteristic damage state has reached. Therefore, transverse matrix damage mode in multidirectional laminates is assumed to be quasibrittle failure, which means that matrix damage accumulates with the increase of the loading [10].

\subsubsection{Fiber/matrix interface debonding}

Interface debonding is mainly caused by in-plane shear stress. As the interface debonds, relative sliding of the discontinuity surfaces makes shear direction behave plastically. Sun and Chen [18] developed a one-parameter plasticity model and Ladeveze and Dantec [16] proposed a meso-scale continuum damage model to describe the nonlinear shear stress-shear strain relation. Similar to Sun and Chen's idea, isotropic hardening behavior in shear direction is postulated to describe the interface damage and the corresponding accumulated shear plastic strain [10].

\subsection{Constitutive theory of irreversible thermodynamics}

Irreversible thermodynamics theory has been employed as a rational framework for the unified formulation of constitutive and evolution equations of elastic-plastic and damage materials $[11,14]$. Free energy characterizing the relation of the internal state variables and their conjugated forces, dissipation function describing the evolution of the internal state variables, and loading surface prescribing the limitation of the elastic region should be postulated in prior. Moreover, using both free energy and dissipation potential to describe the irreversible material behaviors is also called a GSM model [11] in the framework of irreversible thermodynamics.

In this study, associated flow rules are adopted, which assume that damage surface is identical to damage dissipation potential and yield surface is identical to yield dissipation potentials. Details of the free energy and dissipation function will be discussed below. However, it should be mentioned that all the proposed formulation are carried out in the material principal coordinate system, where 1 and 2 represent the directions parallel to and transverse to fiber, respectively.

\subsubsection{Gibbs free energy}

If we consider fiber and transverse directions to be elastic-damage and shear direction to be elastic-plastic with isotropic hardening, the Gibbs free energy $\Gamma$ an be expressed as [10,19]

$\Gamma(\sigma, d, r, \beta)=\Gamma^{\mathrm{e}}(\sigma, d)+\Gamma^{\mathrm{p}}(r)+\Gamma^{\mathrm{d}}(\beta)$,

where the free energy $\Gamma^{\mathrm{e}}$ is the complementary strain energy and may be influenced by the damage. Under the plane stress assumption, $\Gamma^{\mathrm{e}}$ can be expressed as

$$
\begin{aligned}
\Gamma^{\mathrm{e}}(\sigma, d)= & \frac{1}{2}\left[\frac{\sigma_{11}^{2}}{E_{11}^{0}\left(1-d_{11}\right)}+\frac{\sigma_{22}^{2}}{E_{22}^{0}\left(1-d_{22}\right)}\right. \\
& \left.-\frac{2 \nu_{12}^{0}}{E_{11}^{0}} \sigma_{11} \sigma_{22}+\frac{\sigma_{12}^{2}}{G_{12}^{0}}\right]
\end{aligned}
$$

where both $d_{11}$ and $d_{22}$ represent 'fiber breakage damage' and 'matrix cracking damage'. $E_{11}^{0}, E_{22}^{0}, G_{12}^{0}$, and $\nu_{12}^{0}$ are Young's moduli and Poisson's ratio of undamaged lamina. The free energy $\Gamma^{\mathrm{p}}$ and $\Gamma^{\mathrm{d}}$ introduced to describe the effect of the accumulated matrix damage and the accumulated shear plastic strain can be expressed as follows:

$\Gamma^{\mathrm{p}}(r)=\frac{1}{(m+1)} A r^{m+1}$

$\Gamma^{\mathrm{d}}(\beta)=\frac{1}{(n+1)} K \beta^{n+1}$

where $A, k, m$, and $n$ are material constants, $r$ the accumulated shear plastic strain, and $\beta$ the accumulated matrix cracking damage.

According to the thermodynamic formulation, the elastic constitutive equation of the damaged lamina can be obtained by $\varepsilon^{\mathrm{e}}=\partial \Gamma^{\mathrm{e}} / \partial \sigma$. Expressing the equation in 
matrix form, we obtain

$$
\begin{aligned}
\left\{\begin{array}{c}
\varepsilon_{11}^{\mathrm{e}} \\
\varepsilon_{22}^{\mathrm{e}} \\
\varepsilon_{12}^{\mathrm{e}}
\end{array}\right\}= & {\left[\begin{array}{ccc}
\frac{1}{E_{11}^{0}\left(1-d_{11}\right)} & -\frac{\nu_{12}^{0}}{E_{11}^{0}} & 0 \\
-\frac{\nu_{12}^{0}}{E_{11}^{0}} & \frac{1}{E_{22}^{0}\left(1-d_{22}\right)} & 0 \\
0 & 0 & \frac{1}{2 G_{12}^{0}}
\end{array}\right] } \\
& \times\left\{\begin{array}{c}
\sigma_{11} \\
\sigma_{22} \\
\sigma_{12}
\end{array}\right\}
\end{aligned}
$$

Then the conjugate forces corresponding to the internal state variables $d_{11}, d_{22}, r$ and $\beta$ can be derived from Eqs. (3) and (4). Thus we have the following equations:

$$
\begin{aligned}
& Y_{11}=\frac{\partial \Gamma^{\mathrm{e}}}{\partial d_{11}}=\frac{\sigma_{11}^{2}}{2 E_{11}^{0}\left(1-d_{11}\right)^{2}}, \\
& Y_{22}=\frac{\partial \Gamma^{\mathrm{e}}}{\partial d_{22}}=\frac{\sigma_{22}^{2}}{2 E_{22}^{0}\left(1-d_{22}\right)^{2}}, \\
& R=\frac{\partial \Gamma^{\mathrm{p}}}{\partial r}=A r^{m} \\
& B=\frac{\partial \Gamma^{\mathrm{d}}}{\partial \beta}=K \beta^{n} .
\end{aligned}
$$

It is worth noting that the damage conjugated forces $Y_{11}$ and $Y_{22}$ in Eqs. (6) and (7) are also called strain energy density release rate, which are similar to strain energy release rate $G$ in Fracture Mechanics [13].

\subsubsection{Damage surfaces and yield surface}

We postulate that elastic range is bounded by several surfaces and each surface represents the limitation of different failure modes. Damage surfaces and yield surface can be expressed as follows [10].

3.3.2.1. Fiber breakage. Because of the perfect brittle failure behavior in fiber direction, the fiber breakage damage surface $F_{11}$ is assumed to be

$F_{11}=Y_{11}-Y_{11 \mathrm{t}}$,

where $Y_{11 \mathrm{t}}$ is the threshold of fiber damage conjugate force.

3.3.2.2. Matrix cracking. Because of matrix crack developments and accumulations, matrix cracking damage surface can be expressed as

$F_{22}=Y_{22}-\left(Y_{22 \mathrm{t}}+B\right)$,

where $Y_{22 \mathrm{t}}$ is the threshold of matrix damage conjugate force. Furthermore, $B$ represents thermodynamic force associated to matrix cracking damage accumulation as in the case of isotropic hardening stress in the plasticity. By using the normality rule, we have that

$\dot{d}_{22}=\dot{\lambda}_{22} \frac{\partial F_{22}}{\partial Y_{22}}=\dot{\lambda}_{22}$,
$\dot{\beta}=-\dot{\lambda}_{22} \frac{\partial F_{22}}{\partial B}=\dot{\lambda}_{22}$,

where $\dot{\lambda}_{22}$ is the matrix cracking associated multiplier and can be obtained from consistency conditions $F_{22}=0$ and $\dot{F}_{22}=0$ [14]. Eventually, the evolution of $d_{22}$ can be obtained as follows:

$\dot{d}_{22}=\dot{\lambda}_{22}=\frac{\dot{Y}_{22}}{B^{\prime}}$

$B^{\prime}=n K \beta^{n-1}$

3.3.2.3. Fiber/matrix interface debonding. Yield surface in shear direction can be expressed as [10,20]

$F_{12}\left(\sigma_{12}, R ; \dot{\varepsilon}_{12}^{\mathrm{p}}, r\right)=\sigma_{12}-\left(R_{0}+R\right)$,

where $R_{0}$ is the initial yield stress and $R$ is the isotropic hardening stress. By using the normality rule, we have

$\dot{\varepsilon}_{12}^{\mathrm{p}}=\dot{\lambda}_{12} \frac{\partial F_{12}}{\partial \sigma_{12}}=\dot{\lambda}_{12}$

$\dot{r}=-\dot{\lambda}_{12} \frac{\partial F_{12}}{\partial R}=\dot{\lambda}_{12}$

where $\dot{\lambda}_{12}$ is the plasticity associated multiplier and can be obtained from consistency conditions $F_{12}=0$ and $\dot{F}_{12}=0$ [14]. Therefore, elastic-plastic constitutive equation in inplane shear direction can be expressed as follows:

$\dot{\varepsilon}_{12}=\dot{\varepsilon}_{12}^{\mathrm{e}}+\dot{\varepsilon}_{12}^{\mathrm{p}}$,

$\dot{\varepsilon}_{12}^{\mathrm{e}}=\frac{\dot{\sigma}_{12}}{2 G_{12}}$

$\dot{\varepsilon}_{12}^{\mathrm{p}}=\dot{r}=\frac{\dot{\sigma}_{12}}{R^{\prime}}$

$R^{\prime}=m A r^{m-1}$.

\subsection{Principal Damage Model}

Fig. 2 shows the Principal Damage Model proposed by Maa and Cheng [10]. The first column represents the definition of the material principal directions, and the second column represents principal stress-strain relations. Obviously, fiber and transverse directions show softening phenomena beyond damage initiation and no plastic strains occur. However, shear direction behaves elastic-plastically with strain hardening. The third column represents fiber damage relation, matrix cracking damage accumulation, and accumulated shear plastic strain relation. In order to implement the Principal 


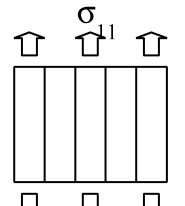

凸 几

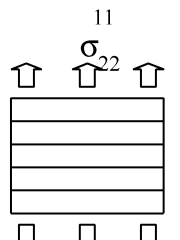

22
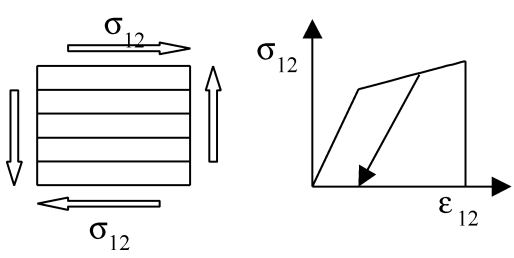

Fig. 2. Schematic illustration of the Principal Damage Model.

Damage Model into finite element program, a further simplification will be discussed below.

\subsubsection{Fiber breakage}

As $Y_{11}$ reaches threshold $Y_{11}$, fiber failure is assumed to occur. $d_{11}$, then, is set to unity.

$d_{11}=\left\{\begin{array}{ll}0 & \text { if } Y_{11}<Y_{11 \mathrm{t}} \\ 1 & \text { otherwise }\end{array}\right.$.

\subsubsection{Matrix cracking}

As $Y_{22}$ reaches its threshold $Y_{22 t}$, matrix cracking initiates. The relation between accumulated matrix damage $d_{22}$ and matrix damage conjugated force $Y_{22}$ can be obtained by integrating Eq. (14)

$d_{22}=\left\{\begin{array}{ll}0 & \text { if } Y_{22}<Y_{22 \mathrm{t}} \\ \left(\left(Y_{22}-Y_{22 \mathrm{t}}\right) / K\right)^{1 / n} & \text { otherwise }\end{array}\right.$.

\subsubsection{Fiber/matrix interface debonding}

Because of strain hardening assumption, shear direction shows hardening without ultimate rupture. To overcome this deficiency, shear absolute failure is assumed to occur as shear stress $\sigma_{12}$ reaches ultimate shear strength obtained from tensile test of angle-ply coupon specimen. When ultimate strength is reached, shear stress is set to zero. Moreover, in order to increase the efficiency of the computation, the non-linear Ramberg-Osgood relationship from the deformation plasticity theory, which allows for a better fit of real stress-strain curves, is adopted to describe the shear hardening in Eqs. (19)-(22) [20]. Taking shear absolute failure into account, we have

$$
\begin{cases}2 \varepsilon_{12}=\frac{\sigma_{12}}{G_{12}^{0}} & \text { if } \sigma_{12}<R_{0}, \\ 2 \varepsilon_{12}=\frac{\sigma_{12}}{G_{12}^{0}}+\alpha\left(\frac{\sigma_{12}}{G_{12}^{0}}\right)^{\mathrm{p}} & \text { if } R_{0} \leq \sigma_{12}<S, \\ \sigma_{12}=0 & \text { otherwise }\end{cases}
$$

where $S$ is the ultimate shear strength, $\alpha$ and $p$ are material constants which can be obtained from curve-fitting to shear stress-shear strain relation.

\section{Experimental and numerical procedures}

\subsection{Materials and tensile test}

AS4/PEEK unidirectional prepregs produced by ICI were lay-up as (1) $[0 / 45 / 90 /-45]_{2 \mathrm{~S}}$ (quasi-isotropic), (2) $\left[0 / 90_{2} / 0\right]_{2 \mathrm{~S}}$ (cross-ply), and (3) $[ \pm 45]_{4 \mathrm{~S}}$ (angle-ply). The prepreg has nominally $61 \mathrm{vol} \%$ of graphite fiber, and is $125 \mu \mathrm{m}$ thick. A matched-die mold was used to form the composite plates following the fabrication conditions recommended by ICI. Circular holes of various sizes were drilled at the specimen center and an ultrasonic C-scan imaging was performed to eliminate any damaged specimens after the machining. The laminate configurations investigated are shown in Table 1.

All tensile tests were performed on an MTS 810 servohydrodynamics machine at room temperature. Tests were conducted under displacement control with a constant rate of $0.2 \mathrm{~mm} / \mathrm{min}$ to avoid dynamic effect. A clip gage was mounted on the center of some notched specimens to monitor the deformation.

\subsection{Finite element model}

Material constitutive equation, Eq. (5) and the internal variable accumulation relations Eqs. (23) and (24) were implemented into commercial finite element code ABAQUS (Version 5.8). User subroutine USDFLD was used to describe the fiber breakage and matrix cracking damages by means of degenerating $E_{11}$ and $E_{22}$. Fig. 3 shows the usage of the subroutine USDFLD. The name of USDFLD is an acronym from USer-Defined FieLD variable. The subroutine is an empty one, which allows the user to program whatever his or her evolution equation of the field (in this case, the damage) variable be. The main program of ABAQUS will call USDFLD at each integration point while forming the element tangent stiffness matrix. In the current implementation, USDFLD calculates $Y_{11}$ and $Y_{22}$ and checks if $Y_{11}=Y_{11 \mathrm{t}}$ and/or $Y_{22}>Y_{22 \mathrm{t}}$ is satisfied. If any damage mode occurs, damage variables will be updated according to Eqs. (23) and (24). The material behavior along 
Table 1

Configurations of laminated plate lay-ups and hole size of AS4/PEEK laminated composite. Thickness $H=2 \mathrm{~mm}, L=100 \mathrm{~mm}$

\begin{tabular}{|c|c|c|c|}
\hline Laminates & $W(\mathrm{~mm})$ & $D(\mathrm{~mm})$ & $D / W$ \\
\hline \multirow[t]{5}{*}[0/45/90/-45]{$_{2 \mathrm{~S}}$} & 20 & 2 & 0.10 \\
\hline & & 3 & 0.15 \\
\hline & & 5 & 0.25 \\
\hline & & 8 & 0.40 \\
\hline & & 10 & 0.50 \\
\hline \multirow[t]{5}{*}[0/45/90/-45]{$_{2 \mathrm{~S}}$} & 30 & 3 & 0.10 \\
\hline & & 6 & 0.15 \\
\hline & & 10 & 0.25 \\
\hline & & 12 & 0.40 \\
\hline & & 15 & 0.50 \\
\hline \multirow[t]{5}{*}[0/90_{2}/0]{$_{2 \mathrm{~s}}$} & 20 & 2 & 0.10 \\
\hline & & 3 & 0.15 \\
\hline & & 5 & 0.25 \\
\hline & & 8 & 0.40 \\
\hline & & 10 & 0.50 \\
\hline \multirow[t]{5}{*}[\pm45]{$_{4 \mathrm{~S}}$} & 20 & 2 & 0.10 \\
\hline & & 3 & 0.15 \\
\hline & & 5 & 0.25 \\
\hline & & 8 & 0.40 \\
\hline & & 10 & 0.50 \\
\hline
\end{tabular}

each direction is affected by the updated damage state accordingly. Because of the explicit scheme adopted by USDFLD, displacement increments should be as small as possible. The increment adopted here is $10^{-3}$.

Because of the plane stress assumption, eight-noded reduced integration plane stress elements, CPS8R, were adopted in this study. Symmetry boundary conditions ( $v=0$ on the $y=0$ centerline and $u=0$ on the $x=0$ centerline) were used to model one-quarter of the laminated plate as shown in Fig. 4. Details of the subroutine formulation can be found in Ref. [10]. The material properties and parameters used in the finite element calculations are listed in Table 2.

\section{Results and discussion}

\section{1. $[ \pm 45]_{4 S}$ laminate}

Fig. 5 shows the comparison between the calculated and measured load-deflection curves of $[ \pm 45]_{4 \mathrm{~S}}$ laminate with hole diameter $D=5 \mathrm{~mm}$ leading up to ultimate failure. The significant non-linearity, which can be attributed to plasticity behavior in the shear direction, appears both in the experimental and finite element results. The predicted damage progression as a function of the applied load is shown in Fig. 6. Observing the finite element predictions, damage initiated from the boundary, near the stress concentration, and propagated along 45-degree from the loading direction. The ultimate failure was dominated by fiber/matrix interface debonding.

Table 3 compares the predicted failure strength with the experimental data of $[ \pm 45]_{4 \mathrm{~S}}$ laminates containing different

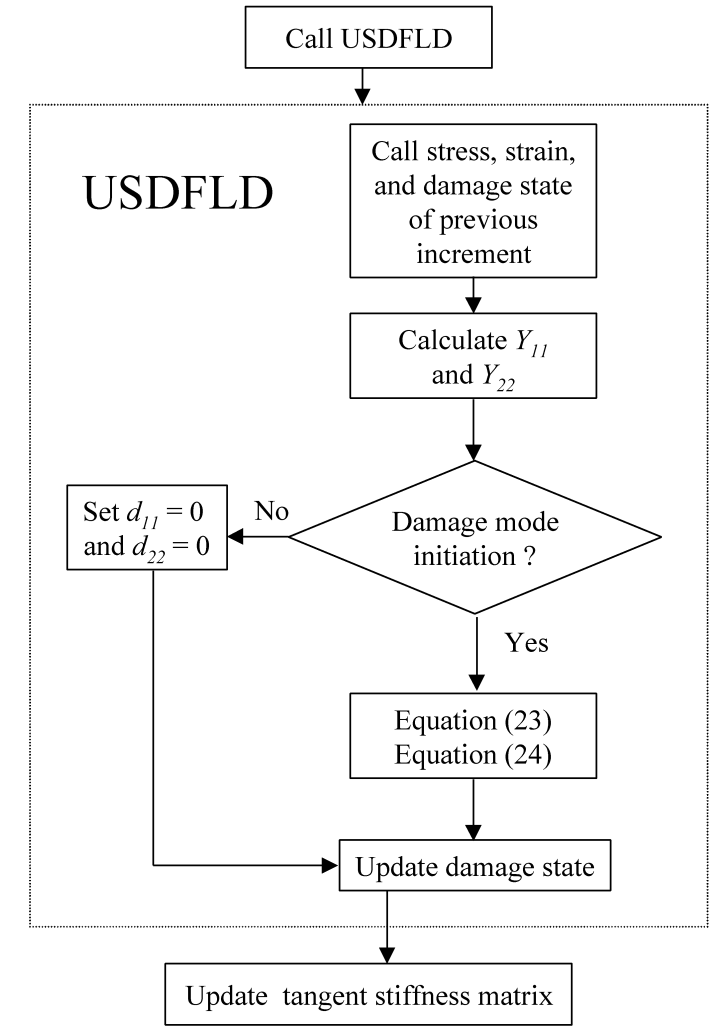

Fig. 3. The flow-chart of user subroutine USDFLD.

hole sizes. The predicted results are consistent with the experimental data except for the $D=3 \mathrm{~mm}$ laminate.

\section{2. $\left[0 / 90_{2} / 0\right]_{2 S}$ and $[0 / 45 / 90 /-45]_{2 S}$ laminates}

The predicted results and experimental data of $\left[0 / 90_{2} / 0\right]_{2 S}$ and $[0 / 45 / 90 /-45]_{2 S}$ laminates are also shown in Table 3. Average errors of 20 and $30 \%$ appear in cross-ply and quasi-isotropic laminates, respectively. Figs. 7 and 8 show the predicted fiber breakage damage pattern in the 0-degree plies of the two laminates. We can observe that as the clipper gage displacement increases, fiber damage extends through out the remaining width and cause the catastrophic failure. It appears that the prefect brittle failure assumption in fiber direction is inadequate to account for the actual damage development. Residual strength remained after fiber breakage. Because the 0degree ply dominated in these two lay-ups, a modified model that can remedy this difficulty will be discussed in Section 6.

\section{Modified Principal Damage Model}

\subsection{Modified model formulation}

Chang et al. [6-8] adopted a micromechanical approach for fiber bundle failure proposed by Rosen [21] to describe the fiber tensile failure in composite materials. It postulates 


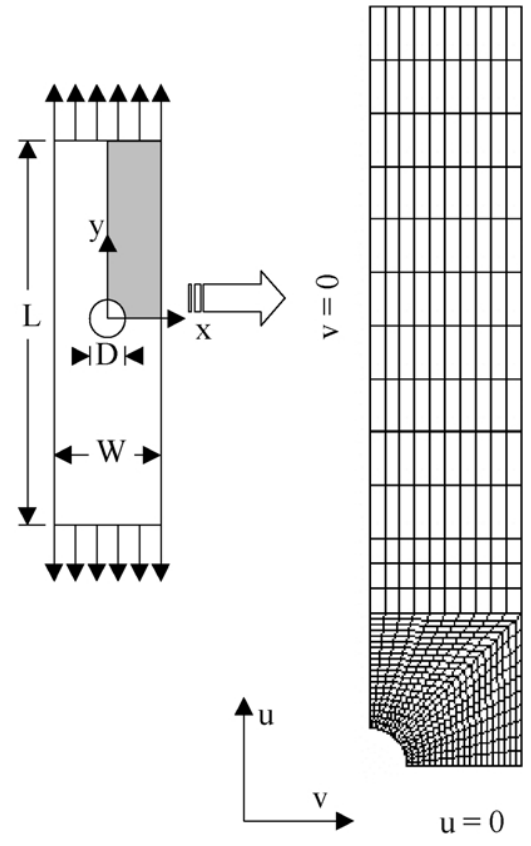

Fig. 4. Finite element mesh for the central hole composite plate.

that longitudinal modulus $E_{11}$ and shear modulus $G_{12}$ degenerate according to the Weibull distribution. Moreover, the modulus degradation function is a function of the fiber failure interaction zone, the shape parameter of the Weibull distribution, and the lamina fiber tensile strength. Although Chang et al. mentioned that their model had the capability to monitor fiber damage, the micromechanical model which needs lot of experimental data to fit the material constants is not practical in structural uses. To overcome the disadvantage of Chang et al. [6-8] and our previous difficult (Eq. (23)), a simple modified fiber damage accumulation rule that can include post-failure of fiber damage behavior is

Table 2

Material properties of laminated composite AS4/PEEK used in the finite element calculation

\section{Moduli}

Undamaged longitudinal Young's modulus, $E_{11}^{0}(\mathrm{GPa})$

Undamaged transverse Young's modulus, $E_{22}^{0}(\mathrm{GPa})$

Undamaged Poisson's ratio, $\nu_{12}^{0}$

Initial in-plane shear modulus, $G_{12}^{0}(\mathrm{GPa})$

Damage parameters

Fiber damage conjugate force threshold, $Y_{11 \mathrm{t}}(\mathrm{MPa})$

Matrix damage conjugate force threshold, $Y_{22 \mathrm{t}}(\mathrm{MPa})$

Matrix damage hardening parameter, $K(\mathrm{MPa})$

Matrix damage hardening parameter, $n$

Fiber damage accumulation parameter, $\gamma$

Plasticity parameters

Shear initial yield stress, $R_{0}(\mathrm{MPa})$

Shear hardening parameter, $\alpha$

Shear hardening parameter, $p$

Shear ultimate strength, $S$ (Mpa)
20 $5.0 \times 10^{-7}$

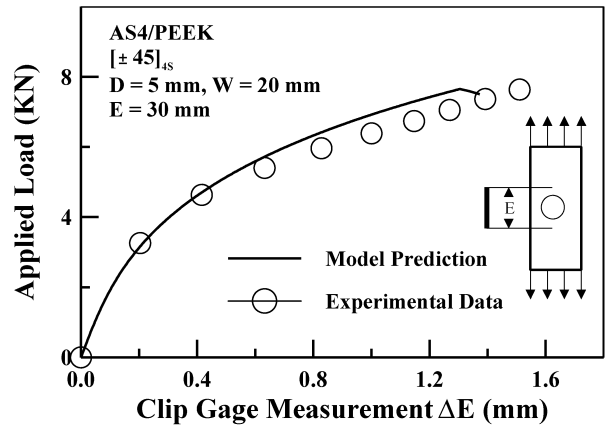

Fig. 5. Comparison between the calculated and the measured loaddeflection curves leading up to ultimate failure. AS4/PEEK $[ \pm 45]_{4 \mathrm{~S}}$ specimen.

proposed to be

$d_{11}=\left\{\begin{array}{ll}0 & \text { if } Y_{11}<Y_{11 \mathrm{t}}, \\ 1-\exp \left(-\gamma\left(Y_{11}-Y_{11 \mathrm{t}}\right)\right) & \text { otherwise }\end{array}\right.$,

where $\gamma$ similar to shape function of Weibull distribution in the fiber bundle theory, is a positive scalar and is called fiber damage accumulation parameter.

Measuring the fiber damage accumulation parameter is extremely difficult to do. Therefore, this value was chosen in an inverse manner to match data from tensile fracture test of the $[0 / 45 / 90 /-45]_{2 \mathrm{~S}}$ specimen with $W=20 \mathrm{~mm}$ and $D=$ $5 \mathrm{~mm}$. It should be mentioned that parameter $\gamma$ can also be obtained from $[0]_{2 \mathrm{~S}}$ specimen with arbitrary hole size. Fig. 9 shows the predicted failure load as a function of fiber damage accumulation parameter $\gamma$. The result indicates that the failure load decreases as parameter $\gamma$ increases. Finally, $\gamma=0.3$ was chosen as the material constant of AS4/PEEK.

Fig. 10 shows fiber damage accumulation pattern in the 0 -degree plies of the $[0 / 45 / 90 /-45]_{2 S}$ laminate. The fiber
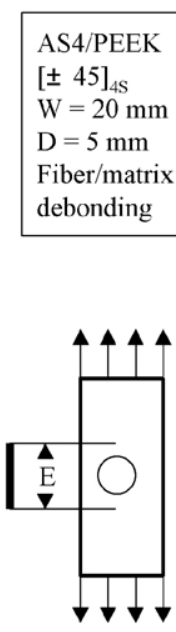

Fig. 6. Illustration of the predicted fiber/matrix debonding damage pattern as a function of the applied load. AS4/PEEK $[ \pm 45]_{4 \mathrm{~S}}$ specimen.
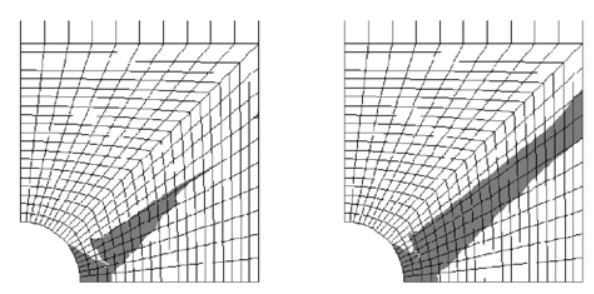

$7.64 \mathrm{KN}$

Failure

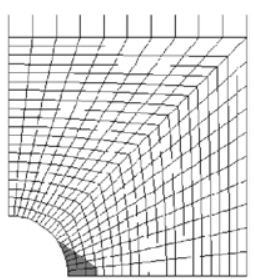

$7.14 \mathrm{KN}$ 
Table 3

Comparison of the failure loads between the experimental data and the FEM predictions. AS4/PEEK, $W=20 \mathrm{~mm}$

\begin{tabular}{lrrl}
\hline Laminates & $\begin{array}{l}D \\
(\mathrm{~mm})\end{array}$ & $\begin{array}{l}\text { Experimental data } \\
(\mathrm{kN})\end{array}$ & $\begin{array}{l}\text { Finite element prediction } \\
(\mathrm{kN})\end{array}$ \\
\hline$[ \pm 45]_{4 \mathrm{~S}}$ & 2 & 12.18 & 10.58 \\
& 3 & 11.12 & 8.65 \\
& 5 & 8.95 & 7.64 \\
{$\left[0 / 90_{2} / 0\right]_{2 \mathrm{~S}}$} & 8 & 6.07 & 6.14 \\
& 10 & 5.33 & 5.14 \\
& 2 & 25.54 & 25.83 \\
& 3 & 23.39 & 14.77 \\
{$[0 / 45 / 90 /-45]_{2 S} \mathrm{~S}$} & 2 & 22.98 & 12.53 \\
& 5 & 21.41 & 10.18 \\
& 3 & 16.32 & 8.74 \\
& 10 & 12.58 & 19.94 \\
& 5 & 15.31 & 13.63 \\
& 8 & 11.67 & 12.00 \\
& 10 & 9.22 & 10.06 \\
\hline
\end{tabular}

damage as a function of clipper gage measured and parameter $\gamma$ are shown. Apparently, the development of fiber damage is suppressed by decreasing the parameter $\gamma$. Moreover, Fig. 11 shows the load-deflection curve of the $[0 / 45 / 90 /-45]_{2 \mathrm{~S}}$ laminate with hole diameter $D=5 \mathrm{~mm}$. It is clear that, the modified model shows good agreements with the experimental data.

\subsection{New model prediction and discussion}

Table 4 shows the comparison of the new model predictions and the experimental data. The predicted results of angle-ply laminates are almost the same compared with the original model, which can be contributed to the minor importance of fiber failure in $[ \pm 45]_{4 \mathrm{~S}}$ laminates. It is worth noting that the prediction of the modified model in $[0 / 45 /$
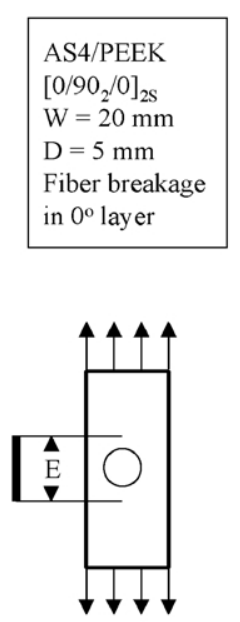

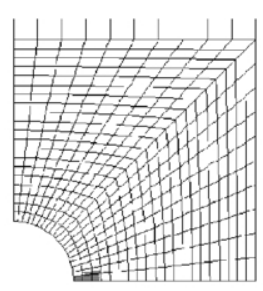

$\Delta \mathrm{E}=0.20 \mathrm{~mm}$

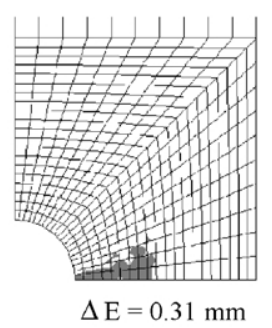

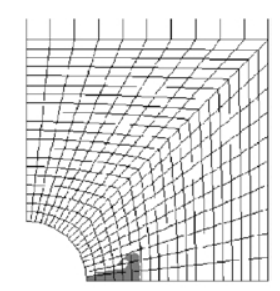

$\Delta \mathrm{E}=0.25 \mathrm{~mm}$

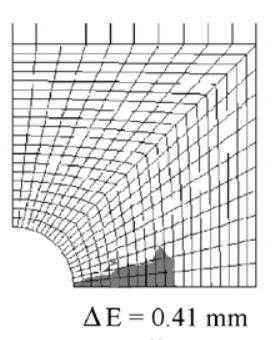

Failure
Fig. 7. Illustration of the predicted fiber breakage damage growth pattern in 0 -degree layer as a function of the clipper gage measurement $\Delta E$. AS4/PEEK $\left[0 / 90_{2} / 0\right]_{2 S}$ specimen.


$\Delta \mathrm{E}=0.30 \mathrm{~mm}$

$\Delta \mathrm{E}=0.40 \mathrm{~mm}$

Failure

Fig. 8. Illustration of the predicted fiber breakage damage growth pattern in 0 -degree layer as a function of the clipper gage measurement $\Delta E$. AS4/PEEK $[0 / 45 / 90 /-45]_{2 \text { s }}$ specimen.

$90 /-45]_{2 S}$ laminates agree well with the experimental data for most of the cases. However, cases for $\left[0 / 90_{2} / 0\right]_{2 \mathrm{~S}}$ laminate still have some errors in the laminates with $D=$ $3 \mathrm{~mm}$ and $D=5 \mathrm{~mm}$.

The errors in $\left[0 / 90_{2} / 0\right]_{2 S}$ laminates indicates that using the present model to predict the softening behavior may result in localization around the stress concentration. However, the so-called localization limiter introduced by Bazant and Pijaudier-Cabot [22] may eliminate the numerical difficulties of the problem. Localization limiter is a regularization procedure; the additional terms are built up from a second gradient approach or from a non-local approach to deal with the material behavior with strain softening properties. Details of the discussion can be seen in Ref. [22].

\subsection{Sensitivity of mesh size}

During investigation, a mesh size sensitivity study was performed to evaluate the effect of mesh size on the predictions of failure strength. Three different meshes

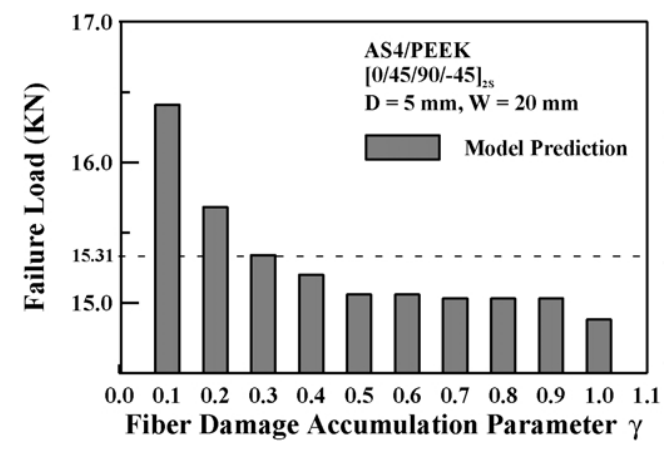

Fig. 9. Illustration of the effect of the fiber damage accumulation parameter $\gamma$ on the predicted failure load. Experimental: $15.31 \mathrm{kN}$. 

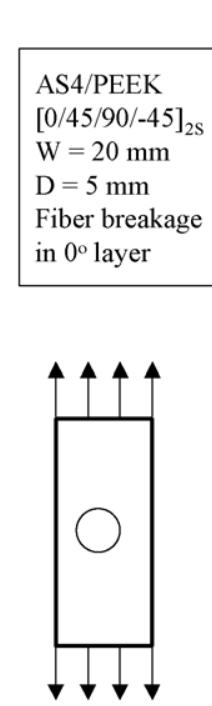

$\gamma=3$

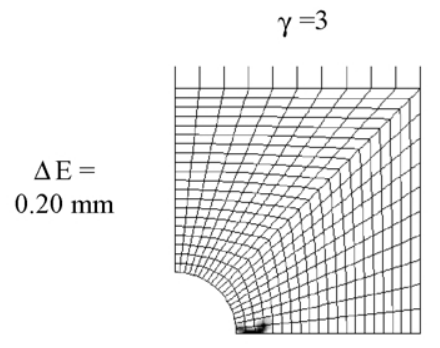

$\Delta \mathrm{E}=$

$0.20 \mathrm{~mm}$
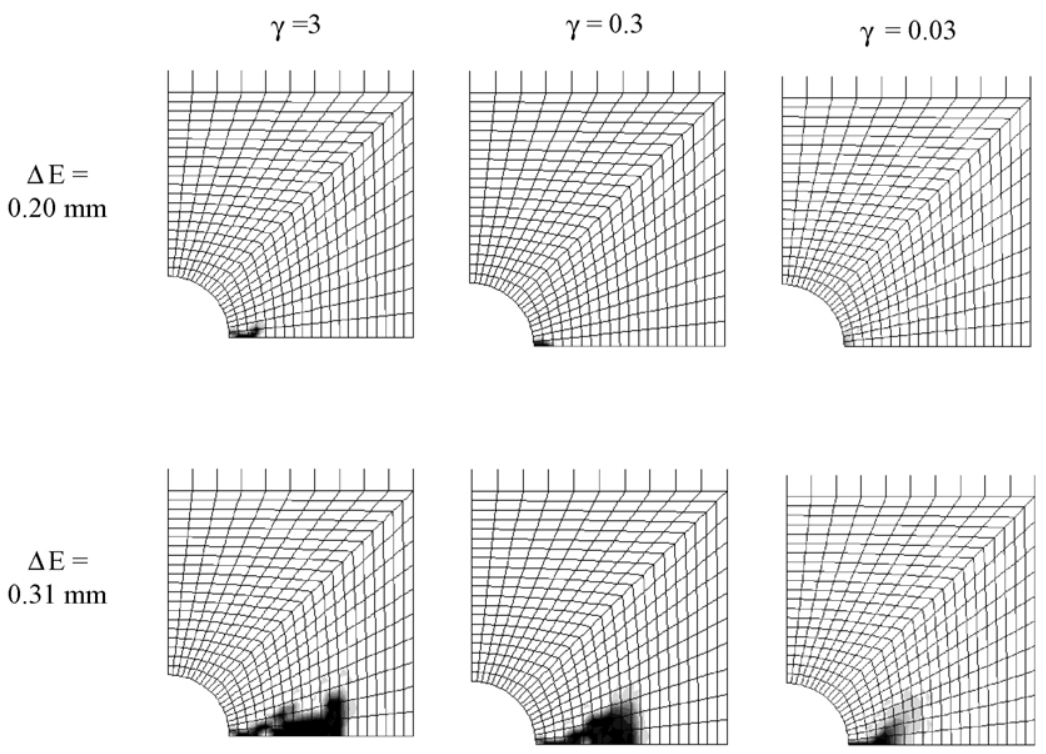

Fig. 10. The fiber damage accumulation pattern as a function of clipper gage measured deflection $\Delta E$ and fiber damage accumulation parameter $\gamma$.

ranged from a coarse (Mesh 1), a medium (Mesh 2) to a fine mesh (Mesh 3) near the hole boundary were generated and shown in Fig. 12.

The comparison of the predicted results of $D=5 \mathrm{~mm}$ and $W=20 \mathrm{~mm}[0 / 45 / 90 /-45]_{2 \mathrm{~S}}$ laminate is shown in Table 5. To sum up, the predictions based on the three meshes were very consistent. It is also worth mentioning that the predicted failure modes in single layer of the laminate were also consistent among the three different meshes.

\subsection{Width effect and comparison with other models}

In order to apply the proposed model to investigate the effect of width with the same diameter/width ratio $(D / W)$ on the failure load, the $W=30 \mathrm{~mm}[0 / 45 / 90 /-45]_{2 \mathrm{~S}}$ laminate with different hole sizes are investigated. Table 6 shows the comparison of the predicted and the experimental results. The results indicate that the proposed model can also predict the failure strength of larger width specimens.

Fig. 13 shows the comparison of the predictions and

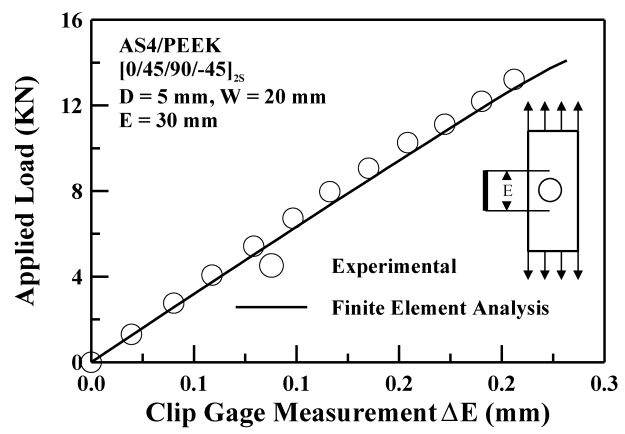

Fig. 11. Comparison between the calculated and the measured loaddeflection curves leading up to ultimate failure. AS4/PEEK $[0 / 45 / 90 /-45]_{2 \mathrm{~S}}$ specimen. experimental results as a function of $D / W$ ratio for laminated plates of $W=20 \mathrm{~mm}$ and $W=30 \mathrm{~mm}$. When the $D / W$ ratio is kept to be constant, e.g. $D / W=0.25$, an increase in the width $W$ leads to a decrease in the failure strength. The width effect or scaling effect on notched composites can be attributed to accumulated damage near the stress concentration around the circular hole. Moreover, the observations are consistent with the results appeared in Ref. [23].

To further validate the model, comparisons of the proposed model with PSC [1] and ASC [2] were performed. Figs. 14 and 15 show the compared results in [0/45/ $90 /-45]_{2 S}$ laminates. The characteristic lengths used in PSC and ASC are obtained from matching the data of the $W=20 \mathrm{~mm}$ and $D=10 \mathrm{~mm}$ laminate. Apparently, the failure strength decreases with an increased hole size in

Table 4

Comparison of failure loads between the experimental results and FEM predictions. AS4/PEEK, $W=20 \mathrm{~mm}$, and $\gamma=0.3$

\begin{tabular}{lrll}
\hline Laminates & $\begin{array}{l}D \\
(\mathrm{~mm})\end{array}$ & $\begin{array}{l}\text { Experimental data } \\
(\mathrm{kN})\end{array}$ & $\begin{array}{l}\text { Finite element prediction } \\
(\mathrm{kN})\end{array}$ \\
\hline$[ \pm 45]_{4 \mathrm{~S}}$ & 2 & 12.18 & 10.61 \\
& 3 & 11.12 & 8.77 \\
& 5 & 8.95 & 7.75 \\
{$\left[0 / 90_{2} / 0\right]_{2 \mathrm{~S}}$} & 8 & 6.07 & 6.23 \\
& 10 & 5.33 & 5.14 \\
& 2 & 25.54 & 27.14 \\
& 3 & 23.39 & 17.55 \\
{$[0 / 45 / 90 /-45]_{2 \mathrm{~S}}$} & 5 & 21.41 & 16.12 \\
& 8 & 16.32 & 12.79 \\
& 2 & 22.98 & 10.99 \\
& 3 & 19.31 & 21.69 \\
& 5 & 15.31 & 17.65 \\
& 8 & 11.67 & 15.34 \\
& 10 & 9.22 & 12.46 \\
\hline
\end{tabular}




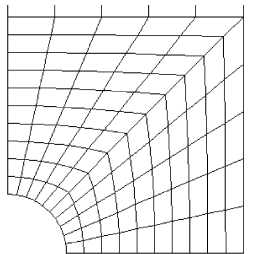

Mesh 1.



Mesh 2.

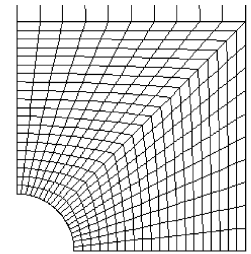

Mesh 3.
Fig. 12. Illustration of three typical finite element meshes used in the mesh size sensitivity study.

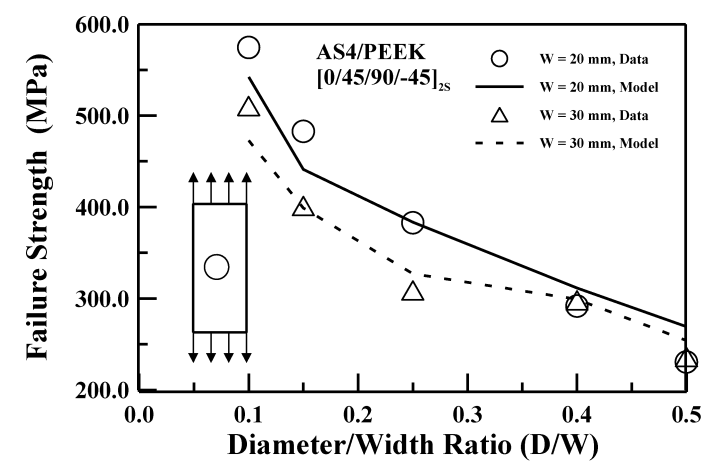

Fig. 13. The comparison of failure strength as a function of a $D / W$ ratio between the predictions and the experimental data. AS4/PEEK, $W=$ $20 \mathrm{~mm}$, and $W=30 \mathrm{~mm}$.

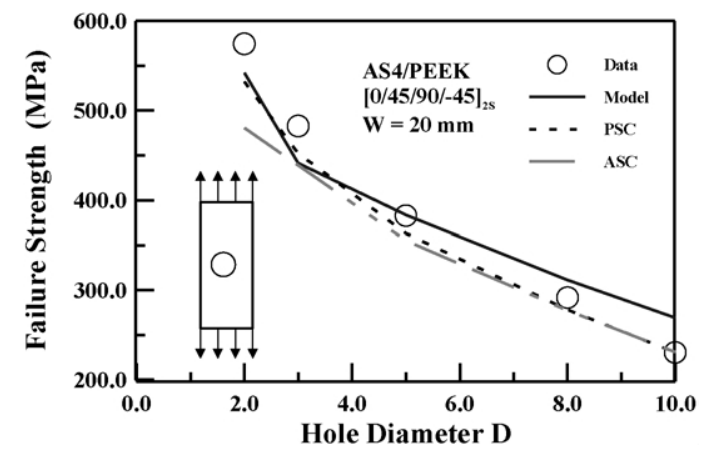

Fig. 14. Comparison of failure strength between the predictions and the experimental data of $[0 / 45 / 90 /-45]_{2 S}$ AS4/PEEK composite laminates. $W=20 \mathrm{~mm}$.

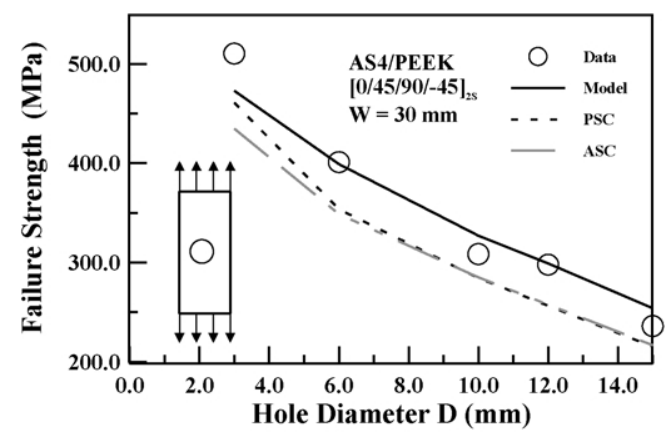

Fig. 15. Comparison of failure strength between the predictions and the experimental data of $[0 / 45 / 90 /-45]_{2 S}$ AS4/PEEK composite laminates. $W=30 \mathrm{~mm}$.
Table 5

Illustration of the effect of mesh size on the predicted failure loads. AS4/PEEK, $D=5 \mathrm{~mm}$, and $W=20 \mathrm{~mm}$. Unit of load is $\mathrm{kN}$

\begin{tabular}{llll}
\hline Laminates & Mesh 1 (coarse) & Mesh 2 (medium) & Mesh 3 (fine) \\
\hline$[0 / 45 / 90 /-45]_{2 \mathrm{~S}}$ & 15.38 & 15.52 & 15.34 \\
\hline
\end{tabular}

Table 6

Comparison of failure loads between the experimental results and FEM predictions. AS4/PEEK, $W=30 \mathrm{~mm}, \gamma=0.3$

\begin{tabular}{llll}
\hline Laminates & $\begin{array}{l}D \\
(\mathrm{~mm})\end{array}$ & $\begin{array}{l}\text { Experimental data } \\
(\mathrm{kN})\end{array}$ & $\begin{array}{l}\text { Finite element prediction } \\
(\mathrm{kN})\end{array}$ \\
\hline$[0 / 45 / 90 /-45]_{2 \mathrm{~S}}$ & 3 & 30.62 & 28.37 \\
& 6 & 24.06 & 23.94 \\
& 10 & 18.51 & 19.62 \\
12 & 17.87 & 17.95 \\
15 & 14.16 & 15.24 \\
\hline
\end{tabular}

every model. Furthermore, as shown in Fig. 15, the proposed model conforms more closely to the experimental data than PSC and ASC. It is because that material constants used in PSC and ASC were obtained from curve-fitting and is dependent on lay-up configurations and notch size. However, the proposed model is based on the irreversible thermodynamics, and has a unified method to obtain the material constants.

\section{Conclusions}

In this study, the Principal Damage Model which combined the Principal Damage concept with GSM model were used in investigating three damage modes in laminated composite materials. After obtaining damage relations, elastic-damage and elastic-plastic constitutive equations were implemented into finite element program to predict the stress-strain relation and the ultimate failure loads of the laminates containing a circular hole. Based on the above investigations, some remarks can be summarized as follows.

1. The post-failure behavior of different failure modes were studied in detail. Contrast to PDM by Chang and Chang [6], the present model provided a unified method, based on irreversible thermodynamics, to obtain the macrostiffness degradation relations and in-plane shear stressshear strain behavior.

2. In order to take the complex geometry into consideration, the commercial finite element code ABAQUS with its powerful user's subroutines was adopted in this study. A particular user's subroutine, USDFLD, was used to define the proposed damage model.

3. The fiber damage behavior plays an important role in the laminated composites failure analysis, and a fiber 
damage accumulation model was proposed to take fiber post-failure behavior into account. By comparing finite element results with experimental data, we proved that the fiber damage accumulation parameter is a material constant.

\section{Acknowledgments}

The authors appreciated the financial support by the National Science Council of Republic of China through the project NSC88-2212-E-002-040.

\section{References}

[1] Whitney JM, Nuismer RJ. Stress fracture criteria for laminated composites containing stress concentration. J Compos Mater 1974;8: 253-65.

[2] Nuismer RJ, Whitney JM. Uniaxial failure of composite laminates containing stress concentrations. ASTM STP 1975;593:117-42.

[3] Backlund J, Aronsson CG. Tensile fracture of laminates with holes. J Compos Mater 1986;20:259-86.

[4] Eriksson I, Aronsson CG. Strength of tensile loaded graphite/epoxy laminates containing cracks, open and filled holes. J Compos Mater 1990;24:456-82.

[5] Afaghi-Khatibi A, Ye L, Mai YW. An effective crack growth model for residual strength evaluation of composite laminates with circular holes. J Compos Mater 1996;30:142-63.

[6] Chang FK, Chang KY. A progressive damage model for laminated composites containing stress concentration. J Compos Mater 1987;21: $834-55$.

[7] Chang FK, Lessard LB. Damage tolerance of laminated composites containing an open hole and subjected to compressive loadings. Part I. Analysis. J Compos Mater 1991;25:2-43.

[8] Shahid I, Chang FK. An accumulative damage model for tensile and shear failures of laminated composite plates. J Compos Mater 1995; 29:926-81.

[9] Lessard LB, Shokrieh MM. Two-dimensional modeling of composite pinned-joint failure. J Compos Mater 1995;29:671-97.

[10] Maa RH, Cheng JH. Principal damage analysis of laminated composite materials. Proc 23rd Natl Conf Theor Appl Mech, Hsinchu, Taiwan 1999;2:64-71

[11] Germain P, Nguyen QS, Suguet P. Continuum thermodynamics. ASME 1983;50:1010-20.

[12] Kachanov LM. Introduction to continuum damage. Mechancis. The Netherlands: Martinus Nijhoff Dordrecht; 1986.

[13] Lemaitre J. A course on damage mechanics. Berlin: Springer; 1996.

[14] Hayakawa K, Murakami S, Liu Y. An irreversible thermodynamics theory for elastic-plastic-damage materials. Eur J Mech A/Solids 1998; 17:13-32.

[15] Simo JC, Ju JW. Strain- and stress-based continuum damage model. I. Formulation. Int J Solids Struct 1987;23:821-40.

[16] Ladeveze P, Dantec EL. Damage modelling of the elementary ply for laminated composites. Compos Sci Technol 1992;43:257-67.

[17] Reifsnider KL. Fatigue of composite materials. The Netherlands: Elsevier; 1991.

[18] Sun CT, Chen JL. A simple flow rule for characterizing nonlinear behavior of fibre composites. J Compos Mater 1989;21:1009-20.

[19] Maugin GA. The thermomechanics of nonlinear irreversible behavior: an introduction. Singapore: World Scientific Publishing; 1999.

[20] Chen WF, Han DJ. Plasticity for structural engineers. New York: Springer; 1988.

[21] Rosen BW. Mechanics of composite strengthening. Fiber composite materials. American Society for Metal; 1965. chapter 3.

[22] Bazant ZP, Pijaudier-Cabot G. Non-local damage: continuum model and localisation instability. J Appl Mech, ASME 1988;55:287-94.

[23] Shaid I, Sun HT, Chang FK. Predicting scaling effect on the notched strength of prepreg and fiber tow-placed laminated composites. J Compos Mater 1995;29:1063-95. 